This dissertation has been

microfilmed exactly as received

$69-19,302$

HUBBARD, Joseph Bunton, 1943-

QUANTUM HARD DISKS AND HARD SPHERES AT HIGH DENSTTIES.

Rice University, Ph.D., 1969

Chemistry, physical

University Microfilms, Inc., Ann Arbor, Michigan 
RICE UNIVERSITY

\section{QUANTUM HARD DISKS AND HARD SPHERES \\ AT HIGH DENSITIES}

by

Joseph Bunton Hubbard

A THESIS SUBMITTED

IN PARTIAL FULFTLLMENT OF THE REQUIREMENTS FOR THE DEGREE OF

Doctor of Philosophy

Thesis Director's signature:

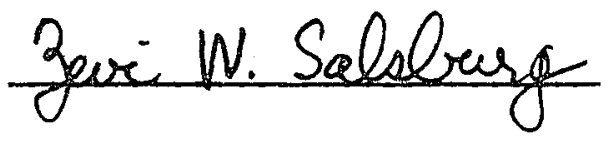

Houston, Texas

June, 1969 


\section{TABLE OF CONTENTS}

Acknowledgements

i

Index of Tables ii

Index of Illustrations iii

I. Introduction 1

II. The Ce11 Cluster Method: An Expansion of the He1mholtz Free Energy $\quad 4$

III. Comparison with Experimental Results: Upper and Lower Bounds on the Ground State Energy 15

Appendix A. The Generalized Faber-Krahn Inequality 21

Appendix B. The Form of the Boundary Conditions on the Wave Function as the Close Packed Density is $\begin{array}{ll}\text { Approached } & 28\end{array}$

References 


\section{ACKNOWLEDGEMENTS}

The author wishes to thank Dr. Zevi $W$. Salsburg for his patience and guidance in this project, Dr. J. E. Kilpatrick for several fruitful suggestions and hints, and Larry Masinter, who was responsible for the computer programming.

The helpful discussions with Dr. W. G. Rudd and Dr. R. F. Curl are gratefully acknowledged, and I especially thank Mrs. D. Butler who typed this thesis.

The author is also indebted to the National Science Foundation and the Robert A. Welch Foundation for the support of this research. 


\section{INDEX OF TABLES}

Table I. The First 20 Eigenvalues for the Single Disk Ce11

Table II. The First 20 Eigenvalues for the Nearest Neighbor Pair of Disks

Table III. The Lowest Eigenvalues for Two Types of Hard Sphere Single Cells 
Figure 1. An Outline of Accessible Configurations for the Nearest Neighbor Pair of Disks in a Triangular Lattice

Figure 2. The Single Particle Cell for Disks in a Triangular Lattice

Figure 3. A Comparison Between the Classical and the Quantum Mechanical Approximations to the Helmholtz Free Energy

Figure 4. A Two Dimensional Closed, Simply Connected Region Each Inner Contour Represents the Wave Function at a Given Value

Figure 5. The Region P of Accessible Configurations Is Shown with Polytopes $\mathrm{P}_{1}$ and $\mathrm{P}_{2}$ where $\mathrm{P}_{1} \subset \mathrm{P} \subset \mathrm{P}_{2}$ with $\mathrm{P}_{2}$ Geometrically similar to $\mathrm{P}_{1}$

Figure 6. A Specific Example which Illustrates the Proof in Appendix B 


\section{INTRODUCTION}

An explanation of the properties of the quantum mechanical solids formed under pressure by $\mathrm{He}^{4}$ and $\mathrm{He}^{3}$ is one of the most interesting unsolved problems in equilibrium statistical mechanics. However, if the He-He repulsive interactions can be approximated by a hard core, one can turn his attention to the problem of calculating the properties of a quantum hard sphere solid as a first step in developing a theory for He. In this paper we will be concerned about the asymptotic behavior of the Helmholtz Free Energy as the density approaches the close-packed density for a system of hard sphere bosons or fermions. The high density limit offers some simplifications (such as localization of the particles) and may be a valuable aid in determining the relative stability of the hexagonal close-packed structure and the face centered cubic structure.

It can be shown that the energy levels of a hard sphere system have the following high density asymptotic form

$$
E_{j} \underset{a \rightarrow \sigma}{\sim} \frac{\mathrm{k}^{j_{h}{ }^{2}}}{2 m(a-\sigma)^{2}},
$$

where $2 \pi \mathrm{h}=\mathrm{h}$ is Planck's Constant, $\mathrm{m}$ is the mass of a sphere, a is the nearest neighbor lattice spacing, $\sigma$ is the hard sphere diameter and $\mathrm{K}^{j}$ is a constant which depends on the lattice configuration. 
To calculate $\mathrm{K}^{j}$ we use the fact that in the limit $\rho \rightarrow \rho_{0}$, the region of accessible configurations is a polytope. ${ }^{1}$ For $N$ spheres under high compression, the region of accessible configurations in the dN-dimensional space $\mathrm{r}_{1},{ }_{\sim}, \ldots,{ }_{\sim} \mathrm{r}_{N}$ contains cavities connected by long narrow tubes. We now specify that our cavities represent different permutations of the spheres for a given close packed lattice configuration. If we denote a close packed configuration by the dN-dimensional vector $x_{\sim}^{(0)}=\left\{R_{\sim}^{0}, R_{\sim}^{0}, \ldots, R_{\sim N}^{0}\right\}$, where $\mathrm{R}_{\sim}^{0}$ is the vector to the ith lattice site, then for a uniform expansion of the lattice in which every particle $i$ moves away from $R_{\sim}^{0}$ a cavity is formed about $x_{\sim}^{(0)}$.

As the external pressure is increased the tubes become narrower and longer so that at high compression we can, to a first approximation, neglect the tubes and discuss the eigenvalue problem for a single cavity. The dN-dimensional space now contains $N$ ! identical disjoint cavities. Each cavity is bounded by curved hypersurfaces (the hypersurfaces are curved because the spheres have curved d-dimensional surfaces), but at very high compression only a small part of each hypersurface serves as the boundary of the allowed region, so we replace the hypersurfaces by their tangent hyperplanes. A proof of this for hard disks and hard spheres is given in Appendix B. Our eigenvalue problem is now that of a resonance cavity in dN-dimensions with the shape of a polytope. 
Alder, Carter and Young ${ }^{2}$ recently determined the first few energy levels in the single particle approximation for hard sphere f.c.c. and h.c.p. lattices, and it would be of interest to know the effects of correlated motion on the relative stability of these two lattices. We have a rough estimate of these effects (see Section III); for both lattices the one particle approximation gives a ground state energy that differs from the exact ground state energy by a factor of about 2.3, but the estimate is not accurate enough to differentiate between the two lattices.

In Section II the cell cluster method is described, and for the case of rigid disks in a triangular lattice, the first 20 energy levels for the single particle cell and the 2-particle cell are given. The Helmholtz Free Energy is then calculated and compared with the classical results.

In Section III we establish upper and lower bounds on the ground state energy for the hard sphere f.c.c. and h.c.p. lattices, and an approximate lower bound for these two lattices is established. 
II. THE CELL CLUSTER METHOD: AN EXPANSION OF THE

HELMHOLTZ FREE ENERGY

We now describe the cell cluster formalism for hard spheres, which generates approximations to the exact $3 \mathrm{~N}$-polytope method described in the Introduction. ${ }^{3}$

First span the N-particle system by a virtual lattice and consider all possible sets of lattice sites which can be connected using nearest neighbor bonds. Classify each set according to the number of sites $n$ and the configuration $t$ and call it a cluster of type $(n, t)$. For each set $(n, t)$ we fix the remaining $N-n$ particles, not associated with the n sites of the cluster, at their lattice positions. We than compute the canonical partition function, $Q_{n, t}$, for the $n$-particles in the cluster and from this obtain the corresponding free energy,

$$
\mathrm{F}_{\mathrm{n}, \mathrm{t}} / \mathrm{k}_{\mathrm{B}} \mathrm{T}=-\ln \mathrm{Q}_{\mathrm{n}, \mathrm{t}}
$$

We then define a series of functions $W_{n, t}$ by means of the following recursion relations:

$$
\begin{aligned}
& \mathrm{W}_{1,1}=\mathrm{F}_{1,1} / \mathrm{k}_{\mathrm{B}} \mathrm{T}, \\
& \mathrm{w}_{\mathrm{n}, \mathrm{t}}=\mathrm{F}_{\mathrm{n}, \mathrm{t}} / \mathrm{k}_{\mathrm{B}} \mathrm{T}-\sum_{\ell=1}^{\mathrm{n}-1} \sum_{\mathrm{S}} \eta_{n, \mathrm{t}}^{\ell, \mathrm{S}} \mathrm{w}_{\ell, \mathrm{s}}, \mathrm{n} \geq 2,
\end{aligned}
$$

where $\eta_{n, t}^{l, s}$ is the number of subclusters of type $(l, s)$ in the cluster $(n, t) .^{4}$ 
For a macroscopic system of $n$ particles, the total free energy may be written

$$
\mathrm{F}_{N} / N k_{B} T=\sum_{n=1}^{N} \sum g(n, t) W_{n, t},
$$

where $\mathrm{Ng}(n, t)$ is the number of different ways the cluster $(n, t)$ can be formed in the system of $\mathrm{N}$ particles. Eq. (II.3) is formally equivalent to the expression

$$
\mathrm{F}_{\mathrm{N}} / \mathrm{Nk}_{\mathrm{B}} \mathrm{T}=-\mathrm{N}^{-1} \ln \mathrm{Q}(\mathrm{N}, \mathrm{V}, \mathrm{T}) .^{5}
$$

The usefulness of Eq. (II.3) depends on the assumption that the dominant terms in the summation over $\mathrm{n}$ are those for which $\mathrm{n}$ is small; that is, we assume that the one particle approximation with additional terms arising from short range correlations accounts for most of the free energy in the system. This assumption appears to be justified in the case of classical hard spheres at high density, as the agreement between the classical cell-cluster theory and machine calculations is very good. ${ }^{6}$ In order to determine the contribution to $\mathrm{F}_{N} / \mathrm{Nk}_{B} \mathrm{~T}$ from each cluster $(n, t)$ for a quantum hard sphere system at high density we must calculate

$$
Q_{n, t}=\sum_{k=0} \exp \left(-\beta E_{k, n, t}\right)
$$

where $k$ indexes a quantum state. The remainder of this section is concerned with the determination of the $\mathrm{E}_{\mathrm{k}, \mathrm{n}, \mathrm{t}}$ for small $\mathrm{n}$ and $\mathrm{k}$.

$$
2
$$


To find $E_{k, n, t}$ we must solve the Schroedinger Wave Equation

$$
\left(-\frac{\hbar^{2}}{2 m} \sum_{j=1}^{n} \nabla_{j}^{2}+E_{k, n, t}\right) \psi_{k, n, t}{ }_{\sim}^{\left(r_{1}, \ldots r_{n}\right)}=0
$$

with the boundary conditions

$$
\psi_{k, n, t}=0,|| r_{\sim j}-r_{i}|-\sigma|=0
$$

where $r_{\sim j}$ is the position vector of the $j$ th particle and $\sigma$ is the hard sphere diameter. The restrictions on $i$ and $j$ in Eq. (II.7) are such that for each particle in $(n, t)$, a nearest neighbor gives rise to a boundary condition. In the close packed limit, the nearest neighbor collision spheres can be approximated by their tangent hyperplanes (see Appendix B) and Eq. (II.7) becomes

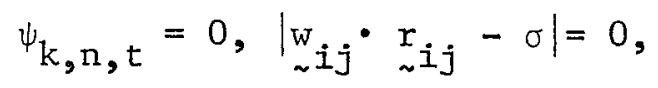

where ${ }_{\sim i j}=R_{i j}^{0} /\left|R_{i j}^{0}\right|$ is a unit vector along the line between lattice sites defined by $\mathrm{R}_{\sim j}^{0}=\mathrm{R}_{\sim j}^{0}-\mathrm{R}_{\sim}^{0}$.

Let $\mathrm{y}_{j}$ be the position vector of the $j$ th sphere center relative to its lattice position $\mathrm{R}_{\mathbf{j}^{\circ}}^{\mathrm{D}}$ Then

$$
r_{i j}=R_{\sim j}^{o}+y_{i j}
$$

and Eq. (A.8) becomes

$$
\psi_{k, n, t}=0,\left|w_{i j} \cdot R_{i j}^{0}+w_{i j} \cdot y_{i j}-\sigma\right|=0 .
$$


Applying the transformation

$$
\begin{aligned}
& {\underset{\sim}{i}}_{i}=\underset{\sim i}{y_{i}} /(a-\sigma), \\
& a=\left|\underset{\sim i j}{R_{i j}^{0}}\right|,
\end{aligned}
$$

and noting that for nearest neighbors

$$
w_{\sim j} \cdot R_{\sim j}^{0}=a,
$$

Eq. (II.10) becomes

$$
\psi_{k, n, t}=0,{\underset{\sim i j}{w}}_{z_{i j}}+1 \mid=0 \text {. }
$$

If we express Eq. (II.6) in terms of the $z_{i}$, we obtain (for $d=3$ )

$$
\begin{gathered}
\left((a-\sigma)^{-2} \frac{\hbar^{2}}{2 m} \sum_{j=1}^{n}\left[\frac{\partial^{2}}{\partial x_{1, j}^{2}}+\frac{\partial^{2}}{\partial x_{2, j}^{2}}+\frac{\partial^{2}}{\partial x_{3, j}^{2}}\right]-E_{k, n, t}\right) \psi_{k, n, t}=0 \\
\underset{\sim j}{z_{j}}=x_{1, j} \hat{i}+x_{2, j} \hat{j}+x_{3, j} \hat{k},
\end{gathered}
$$

where $\hat{i}, \hat{j}, \hat{k}$ are mutually orthogonal unit vectors. The boundary conditions for Eq. (II.14) are

$$
\psi_{k, n, t}\left(z_{1}, \ldots z_{\sim n}\right)=0,\left|w_{i j} \cdot z_{i j}+1\right|=0 .
$$


$\psi_{k, n, t}$ represents the wave function of a point mass m moving freely inside a $3 n$ dimensional polytope, the potential becoming infinite on the boundary surface of the polytope.

If we are to solve Eq. (II.14) using the Method of Linear Variations ${ }^{7}$, we must construct a set of functions that are linearly independent of one another, and that satisfy the boundary conditions in Eq. (II.15). Each member of such a set could be constructed by multiplying the product of the boundary functions $\left(w_{i j} \cdot Z_{i j}+1\right)$ by a polynomial, $M_{\ell}$, which is linearly independent of any other $\mathrm{M}_{\ell}$ we use. The use of polynomial wavefunctions enables us to analytically calculate matrix elements with greater accuracy than if we used more complex functions and had to integrate numerically to obtain matrix elements.

As an example, take the case $n=2$ for rigid disks in a two-dimensional triangular lattice (see Figure 1 and Figure 2); there is only one cluster of this type with $g(2,1)=3$, and there are eleven boundary conditions. We shall choose the coordinate system so that the origin is the lattice site of particle 1 , and the coordinate system for particle 2 is obtained by translating the coordinate system for 1 in the positive direction along the $x_{1,1}$ axis. For simplicity in notation, let $x_{1,1}=x_{1} ; x_{1,2}=x_{2}$; $x_{2,1}=x_{3} ; x_{2,2}=x_{4}$, so that the interior and boundary of this fourdimensional polyhedron are described by 


$$
\begin{array}{l|l}
-\frac{1}{\sqrt{3}} x_{1}-x_{2}+\frac{2}{\sqrt{3}} \geq 0, & -\frac{1}{\sqrt{3}} x_{3}-x_{4}+\frac{2}{\sqrt{3}} \geq 0, \\
x_{1}+1 \geq 0, & 1-x_{3} \geq 0, \\
x_{2}+\frac{1}{\sqrt{3}} x_{1}+\frac{2}{\sqrt{3}} \geq 0, & x_{4}+\frac{1}{\sqrt{3}} x_{3}+\frac{2}{\sqrt{3}} \geq 0, \\
x_{2}-\frac{1}{\sqrt{3}} x_{1}+\frac{2}{\sqrt{3}} \geq 0, & x_{4}-\frac{1}{\sqrt{3}} x_{3}+\frac{2}{\sqrt{3}} \geq 0
\end{array},
$$

The wave function, $\psi\left(x_{1}, x_{2}, x_{3}, x_{4}\right)$, must be identically zero when any one of the above inequalities becomes an equality. The most obvious way to insure this is to take

$$
\psi\left(x_{1}, x_{2}, x_{3}, x_{4}\right)=\sum_{j=0}^{\infty} a_{j} \phi_{j}\left(x_{1}, x_{2}, x_{3}, x_{4}\right),
$$

where the $\phi_{j}$ are formed by taking the product of the eleven boundary functions in Eq. (II.16) and multiplying the result by a modifying polynomial $M_{j}\left(x_{1}, x_{2}, x_{3}, x_{4}\right)$. If we minimize the energy with respect to the $a_{j}$ we obtain the Secular Determinant whose roots are the eigenvalues we seek. 
Let us write $\phi_{j}=M_{j} D$, where $D$ is the product of boundary functions and $\mathrm{M}_{j}$ is the modifying polynomial. If we wish to approximate the ground state wave function which has no nodes, the natural choice for $M_{j}$ is to take $M_{j}=D^{j}$, which gives a Lagrange Expansion for $\psi$. Such a choice is not practical because of the number of terms in the $\phi_{j}$. In the above example, $D$ has 115 terms, $D^{2}$ has 1181 terms, and $D^{4}$ has well over a half million terms.

A more practical method, and the one that was used, is to take $M_{j}=x_{1}{ }^{N_{1}} x_{2}{ }^{N_{2}} x_{3}^{N_{3}}{ }_{x_{4}}^{N_{4}}$ with the restriction $\sum_{i=1}^{4} N_{i} \leq 4$, where $N_{i}$ is either 0 or a positive integer $\leq 4$. There are 70 linearly independent $\phi_{j}$ that can be constructed using this scheme and all 70 of these were used. Convergence to the lowest eigenvalue was good enough so that more sophisticated variation procedures were considered unnecessary. A similar procedure was used for the case of a single disk surrounded by fixed nearest neighbors (see Figure 2 and Figure 6). We chose $\mathrm{M}_{\mathrm{j}}=\mathrm{x}_{1}{ }^{\mathrm{N}_{1}} \mathrm{x}_{2}{ }^{\mathrm{N}_{2}}\left(\mathrm{x}_{1}=0\right.$ and $\mathrm{x}_{2}=0$ being the origin $)$ with $\mathrm{N}_{1}+\mathrm{N}_{2} \leq 20$, and 91 of the 191 possible combinations for $M_{j}$ were used.

Table I and Table II list the first 20 eigenvalues for the 1-disk and 2-disk cases, respectively. The eigenvalues are given in units of $\frac{\hbar^{2}}{2 m}(a-\sigma)^{-2}$, where $m$ is the mass of a disk, a is the nearest neighbor lattice distance, and $\sigma$ is the diameter of a disk. An accurate estimate of the error in each of the eigenvalues is difficult to obtain. ${ }^{8}$ One reason is that in our choice of wave functions we have restricted ourselves to a single function formed by taking the product of the boundary functions, 
and we also have those functions which are zero on at least one coordinate axis. Linear combinations of these functions will possess many different symmetries, so that the inclusion of an additional basis function may or may not contribute to the lowering of a given eigenvalue. In addition, it is possible that some wavefunctions cannot be described by any linear combination of our basis functions, in which case we will have omitted those eigenvalues. The latter possibility is remote as long as we consider only the first $\ell$ eigenvalues using $\mathbb{N}$ basis functions where $\ell$ is less than $\frac{1}{3} N$. We tested this assumption by observing how a given eigenvalue changed as we varied N. If $l \leq 20$, there were only small changes in these eigenvalues for $N>30$. This was true for both the single and two particle cases. Since the maximum value of $\mathrm{N}$ is 91 for the single particle case and 70 for the two particle case, it seemed unlikely that some linear combination of 40 or 50 basis functions in addition to the first 30 would not give a reasonable approximation to the lth wavefunction. The diagonalization routine used resulted in eigenvalues that were accurate to 8 significant figures, so that this source of error could be disregarded. By examining the converging sequence for each of 
the first 20 eigenvalues, we have concluded that our eigenvalues for the two particle case are accurate to four significant figures, and our eigenvalues for the single particle case are accurate to five significant figures. Our main criterion was that the lowest eigenvalue was accurate to s significant figures if there was no change in the sth significant figure upon the addition of the last twelve basis functions.

The main drawback of the method we used was the amount of time spent in programming and running the problem on the Rice Computer. The two particle case, for example, required nearly 140 hours of computer time to be run and checked once. We hope that the time factor can be significantly reduced by making efficient use of a more modern, high speed machine.

In Table III we present the results obtained by Alder, Carter and Young ${ }^{9}$ for the two types of close packed 3-dimensional lattices. The first few levels for the single particle cells corresponding to the hexagonal close packed (h.c.p.) lattice and the face centered cubic (f.c.c.) lattice are given in units of $\frac{\hbar^{2}}{2 m}(a-\sigma)^{-2}$, where $m, a$, and $\sigma$ have the same meaning as in the 2-dimensional cases. 
If we return to the first entries in Table I and Table II, we see that for rigid disks, using the cell cluster formalism, the ground state energy per particle is given by

$$
E_{\circ}=\frac{h^{2}}{2 m(a-\sigma)^{2}}[5.3667+1.881] \text {, }
$$

or

$$
E_{o}=\frac{h^{2}}{2 m(a-\sigma)^{2}}[7.248]
$$

The second term in Eq. (II.18) is the cell cluster correction for pair correlation.

We now present a comparison between the quantum approximation to the Helmholtz Free Energy for rigid disks and the corresponding classical approximation. We shall assume that the density of the system is such that corrections for the curvature of the disks can be neglected. The quantum Helmholtz Free Energy is given by

$$
\begin{aligned}
\mathrm{F}_{\mathrm{q}} / \mathrm{Nk}_{\mathrm{B}} \mathrm{T} \approx-\ln \left[\mathrm{e}^{-5.3667 \mathrm{~B}}+2 \mathrm{e}^{-13.599 \mathrm{~B}}+\ldots\right] \\
-\quad-3\left(\ln \left[\mathrm{e}^{-11.360 \mathrm{~B}}+\mathrm{e}^{-18.352 \mathrm{~B}}+\ldots\right]\right. \\
\left.-2 \ln \left[\mathrm{e}^{-5.3667 \mathrm{~B}}+2 \mathrm{e}^{-13.599 \mathrm{~B}}+\ldots\right]\right)
\end{aligned}
$$

where

$$
-B=\frac{h^{2}}{8 \pi^{2} m_{B} T(a-\sigma)^{2}}
$$


Now $v / v_{0}=a^{2} / \sigma^{2}$, so that

$$
B=\frac{h^{2}}{\sigma^{2} 8 \pi^{2} m k_{B} T\left(\left[\frac{v}{v_{0}}\right]^{\frac{1}{2}}-1\right)^{2}} \underset{v_{0} r_{0}}{\longrightarrow} \frac{h^{2}}{\sigma^{2} 2 \pi^{2} m k_{B} T\left(\frac{v}{v_{0}}-1\right)^{2}} .
$$

The classical Helmholtz Free Energy is given by ${ }^{10}$

$$
\mathrm{F}_{C} / N k_{B} \mathrm{~T} \approx 2 \ln \left(\frac{\lambda}{\sigma}\right)-2 \ln \left(\frac{\mathrm{V}}{\mathrm{v}_{0}}-1\right)+0.1328+\mathrm{O}\left(\frac{\mathrm{V}}{\mathrm{v}_{\mathrm{o}}}-1\right)
$$

where

$$
\lambda=\left(\frac{h^{2}}{2 \pi m k_{B} T}\right)^{\frac{1}{2}}
$$

is the mean thermal wavelength. We write Eq. (II.23), neglecting terms of $\operatorname{order}\left(\left[v / v_{0}\right]-1\right)$, as

$$
\mathrm{F}_{\mathrm{C}} / \mathrm{Nk} \mathrm{B}_{\mathrm{B}} \mathrm{T} \approx \ln \left[\mathrm{e}^{0.1328} \pi \mathrm{B}\right]
$$

and compare Eq. (II.25) with Eq. (II.20) for different values of B (see Fig. 3). If we fix $v / \bar{v}_{0}$ at some value very close to 1 , then for disks of a given size and mass, $B$ is proportional to $1 / T$. We expect the quantum results to be appropriate for large $B$ and the classical approximation to hold for small B.

For $\mathrm{B}<.6$ the higher energy levels become important and so Eq. (II.20) becomes a poor approximation for $\mathrm{F}_{\mathrm{q}} / \mathrm{Nk}_{\mathrm{B}} \mathrm{T}$. If we had the entire eigenvalue spectrum, $F_{q} / N k_{B} T$ would approach $F_{C} / N k_{B} T$ as $B \rightarrow 0$ at constant v. 
III. COMPARISON WITH EXPERIMENTAL RESULTS: UPPER AND LOWER BOUNDS ON THE GROUND STATE ENERGY

In this section we compare the results we obtain for $E_{0}$, the ground state energy per particle, with experimental data.

The following scheme was employed by $\mathrm{R}$. Cole ${ }^{11}$ to relate pressurevolume data to the energy levels of a dense many-body system whose atoms possess a hard-core repulsive potential. It is assumed that in the close packed limit each particle of diameter $\sigma$ is confined to move in a region with characteristic dimension $R$, the mean particle spacing. By the Heisenberg principle, each has a momentum $\propto h(R-\sigma)^{-1}$, and a kinetic energy $\propto h^{2} / 2 m(R-\sigma)^{-2}$. Expressed in terms of densities, the ground state energy per particle may be written

$$
E_{0} \underset{\rho \rightarrow \rho_{0}}{\sim} A \frac{\hbar^{2}}{2 m}\left(\rho^{-\frac{1}{3}}-\rho_{0}^{-\frac{1}{3}}\right)^{-2},
$$

where $\rho_{0}$ is the ultimate density and $A$ is a constant. This result is independent of statistics, since the particles are localized in a lattice and are therefore distinguishable. For low temperatures and high densities it is assumed that the system energy levels are so closely spaced that Eq. (III.1) still holds if we replace $E_{o}$ with $E_{\text {. Now consider a }}$ non-singular attractive potential $\mathrm{V}^{\prime}$, in addition to the hard-core potential. It is reasonable to determine the system energy levels by considering $V^{\prime}$ as a perturbation and carrying out a perturbation expansion using as unperturbed states the hard sphere states. To first 
order in $V^{\prime}$ the energy per particle can be written

$$
E=E_{h . s_{0}}+\left\langle\psi\left|V^{\prime}\right| \psi\right\rangle,
$$

where $|\psi\rangle$ is the hard sphere state. Cole considered two systems with the same $V^{\prime}$ and $\rho_{0}$ but with different masses, $M_{1} m_{u}$ and $M_{2} m_{u}$ (m $m_{u}$ is the atomic mass unit). He then took the volume derivative of

$$
E_{2}(\rho)-E_{1}(\rho)=\frac{M_{1}-M_{2}}{M_{1} M_{2}} E^{*}(\rho) \text { h.s. }
$$

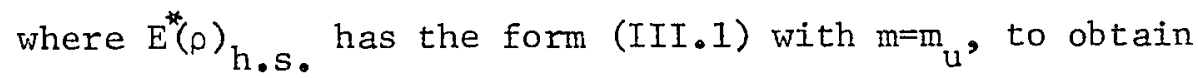

$$
\rho^{-\frac{1}{3}}=\rho_{0}^{-\frac{1}{3}}+\left(\frac{2 A}{3} \frac{\hbar^{2}}{2 m_{u}}\right)^{\frac{1}{3}} \rho^{\frac{2}{9}} \rho_{0}^{-\frac{1}{3}}
$$

where

$$
\begin{aligned}
& P_{\circ}=\frac{M_{1} M_{2}}{M_{1}-M_{2}}\left[P_{2}(\rho)-P_{1}(\rho)\right]_{T=0}, \\
& P_{i}(\rho)=-N \frac{\partial E_{i}(\rho)}{\partial V}=\rho^{2} \frac{\partial E_{i}(\rho)}{\partial \rho},
\end{aligned}
$$

$P_{2}(\rho)$ and $P_{1}(\rho)$ being the external pressure on systems 2 and 1 . A plot of $\rho{ }^{\frac{2}{9}} \mathrm{P}_{0}^{-\frac{1}{3}}$ against $\rho^{-\frac{1}{3}}$ gave (approximately) a straight line whose slope gave $A$ and whose $\rho^{-\frac{1}{3}}$ intercept gave $\rho_{0}$. Experimental data for the $\mathrm{He}^{3}-\mathrm{He}^{4}$ pair resulted in $\mathrm{A}=15.7 \pm 2.0$.

We will now show that the Faber-Krahn Inequality (see Appendix A) generates an expression for $\mathrm{E}_{\mathrm{o}}$ which is asymptotically equivalent to Eq. (III.I) 
In Eq. (A.19), if we examine the large $l$ asymptotic form of $\mathrm{K}_{\mathrm{E}, \frac{\ell-2}{2}, 1}^{2}$ and $\Gamma(1+\ell / 2)^{-\frac{2}{\ell}}$ we obtain

$$
\begin{aligned}
& \mathrm{K}^{2}, \frac{\ell-2}{2}, 1 \sim \ell^{2} / 4, \\
& \Gamma(1+\ell / 2)^{-\frac{2}{\ell}} \sim 2 \text { e } \ell,
\end{aligned}
$$

so that Eq. (A.19), for large $\ell$, may be written

$$
\begin{gathered}
E_{0} \sum_{p \rightarrow p_{0}} \frac{\hbar^{2}}{2 \mathrm{~m}} \frac{\mathrm{de} \pi}{2} \mathrm{v}^{-\frac{2}{l},} \\
\ell=\mathrm{d} N,
\end{gathered}
$$

where $\mathrm{N}$ is the number of particles in the system.

We use an asymptotic expression for $V$, the classical configurational integral, which was derived by Salsburg et al.: ${ }^{6}$

$$
\begin{aligned}
& \mathrm{V}^{\frac{1}{N}} \underset{\alpha \rightarrow 0}{\sim} \sigma^{\mathrm{d}}\left(\frac{\alpha}{1-\alpha}\right){ }_{i=0}^{\mathrm{d}} \prod_{i}^{-\gamma_{i} \alpha^{i}}, \\
& \alpha=1-\left(\rho / \rho_{0}\right)^{\frac{1}{d}}=(a-\sigma) / a,
\end{aligned}
$$

where $\gamma_{1}, \gamma_{2} \ldots$ are constants which account for the curvature of the hard spheres. In particular, for $d=3$ and for the f.c.c. and h.c.p. lattices: 


$$
\begin{array}{ll}
\text { h.c.p. } & \gamma_{0}=C=-1.515, \\
\gamma_{1}=D & =-1.326, \\
\text { f.c.c. } \quad \gamma_{0}=C=-1.523, & \gamma_{1}=D=-1.344 .12,13
\end{array}
$$

Thus Eq. (III.7) may be written

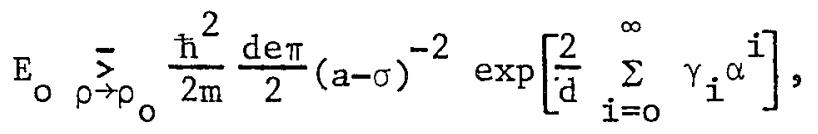

$$
\text { E } \sum_{\rho \rightarrow p} \frac{\hbar^{2}}{2 \mathrm{~m}} \frac{\mathrm{de} \pi}{2}(a-\sigma)^{-2} \mathrm{e}^{\frac{2 \gamma}{\mathrm{d}}} \mathrm{o}\left[1+\frac{2}{\mathrm{~d}} \gamma_{1} \alpha+\left(\frac{2 \gamma_{2}}{\mathrm{~d}}+\frac{2 \gamma_{1}^{2}}{\mathrm{~d}^{2}}\right) \alpha^{2}+0\left(\alpha^{3}\right)\right] \text {. }
$$

Eq. (III.11) is an asymptotic Laurent Expansion for $\mathrm{E}_{\mathrm{o}}$, the dominant term for small $\alpha$ being the first term, which has a second order pole at $\alpha=0$. In order to compare Eq. (III.11) or Eq. (III.10) with Eq. (III.1) we must consider the case $d=3$ for the h.c.p. or f.c.c. lattice. First consider that for both close packed lattices

$$
\begin{aligned}
& (a-\sigma)^{-2}=\left(\rho^{-\frac{1}{3}}-\rho_{0}^{-\frac{1}{3}}\right)^{-2} 2^{\frac{1}{3}}, \\
& \rho_{0}^{-1}=v_{0}=\sigma^{3} 2^{-\frac{1}{2}} .
\end{aligned}
$$


We now compare the first term in Eq. (III.11) with Eq. (III.1), using $\gamma_{0}=-1.515:$

$$
\begin{aligned}
& E_{0} \geq \frac{\hbar^{2}}{2 m \rho}\left(\rho^{-\frac{1}{3}}-\rho_{0}^{-\frac{1}{3}}\right)^{-2}\left[\frac{3 e \pi}{2} 2^{\frac{1}{3}} \exp (-1.008)\right], \\
& E_{0} \geq \underset{\rho \rightarrow \rho}{\geq} \frac{\hbar^{2}}{2 m}\left(\rho^{-\frac{1}{3}}-\rho_{0}^{-\frac{1}{3}}\right)^{-2}(5.94) .
\end{aligned}
$$

Eq. (III.14) establishes an approximate asymptotic lower bound on $\mathrm{E}_{\mathrm{O}}$ in the high density limit. Because the accuracy of $\mathrm{K}^{\mathrm{O}}$ is determined by $\gamma_{0}$ which is probably correct to within a few per cent ${ }^{12}$ we feel that Eq. (III.14) is very close to the best asymptotic high density lower bound on $\mathrm{E}_{\mathrm{O}}$ that can be obtained using the Faber-Krahn Inequality. The experimental densities were such that $\rho / \rho_{0}$ ranged from .14 to .38 which is considerably less than 1 . Thus we would not expect Eq. (III.14) to be applicable in this range. From Eq. (III.8), Eq. (III.9) and Eq. (III.10) we see that since $\gamma_{1}$ is negative, if we include terms of order $\alpha$ in Eq. (III.10) for any $\rho / \rho_{0}<1$, we will obtain a lower bound on $\mathrm{E}_{\mathrm{o}}$ which is less than that given by $\mathrm{Eq}$. (III.14).14,15

If we use a lower bound for $\gamma_{0}\left(f_{0} c_{0} c_{0}\right),-2.23$, which gives an upper bound for $\mathrm{V}$ in the high density limit, ${ }^{16}$ we obtain

$$
E_{0} \geq \underset{\rho \rightarrow \rho_{0}}{ } \frac{\hbar^{2}}{2 m}\left(\rho^{-\frac{1}{3}}-\rho_{0}^{-\frac{1}{3}}\right)^{-2}(1.63)
$$


Eq. (III.15) establishes a rigorous lower bound on $E_{0}$ in the high density limit; we shall now establish an upper bound for $\mathrm{E}_{\mathrm{O}}$ by considering the high density single particle cell, where the cell is so small that the spheres cannot contact one another. ${ }^{17}$ Each cell has the shape of a rhombic dodeahedron (f.c.c.) or an icosahedron (h.c.p.), the shortest distance between the cell's center and a facet being equal to $\frac{1}{2}(a-\sigma)$, where $a$ is the distance between nearest neighbor lattice sites. Alder, Carter, and Young have found the first few energy levels for each of these cells, and the lowest level gives the upper bound for $\mathrm{E}_{\mathrm{O}}$.

$$
E_{0} \underset{\rho \rightarrow \rho_{0}}{\sum_{j}} \frac{\hbar^{2}}{2 m}\left(\rho^{-\frac{1}{3}}-\rho_{0}^{-\frac{1}{3}}\right)^{-2}(27.0),
$$

which holds for both h.c.p. and f.c.c. cells. 18

We obtain the Lennard-Jones-Devonshire (L.J.D.) expression for $E_{0}$ if we consider the single particle cell whose shortest distance between center and a facet equals $(a-\sigma)$. This cell gives $A=6.73$, which

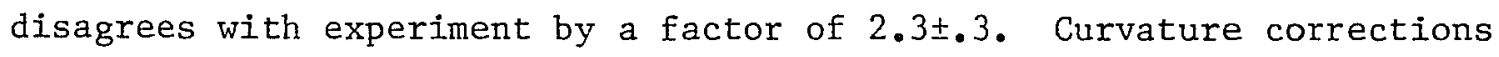
for the L.J.D. polyhedral cell would result in a value for $A$ which is less than 6.73 since the polyhedral cell is enclosed by the exact cell. Thus it appears that the L.J.D. approximation to $E_{0}$ for a system of quantum hard spheres is rather poor, correlations between two or more particles increasing the L.J.D. $E_{0}$ by a factor of about 2.3. This conclusion is based on the assumptions that the attractive part of the He-He potential contributes to the first order perturbation energy only, that the repulsive portion of the potential can be closely approximated by a hard core, and that spin alignment can be neglected at temperatures of about $2^{\circ} \mathrm{K} .19,20$ 
APPENDIX A

The generalized Faber-Krahn Inequality enables us to establish a lower bound on the lowest energy level of a system of d-dimensional hard spheres at high density. A proof of the inequality for two dimensions is given by Garabedian ${ }^{21}$, and the generalization presented below, though not obvious, is straightforward.

Consider an $\ell$-dimensional region $\mathrm{P}$ with boundary $\delta \mathrm{P}$, and consider the solution to the differential equation

$$
\begin{aligned}
& \left(\nabla_{\ell}^{2}+\lambda_{P, \ell}^{0}\right) u_{1}\left(x_{1} \ldots x_{\ell}\right)=0, \\
& \nabla_{\ell}^{2}=\sum_{i=1}^{\ell} \partial^{2} / \partial x_{i}^{2}, \\
& u_{\left.1\right|_{\delta P}=0 .}
\end{aligned}
$$

$\lambda_{\mathrm{P}, \ell}^{\mathrm{O}}$ is the lowest eigenvalue associated with Eq. (A.1) and $u_{1}$ is the corresponding eigenfunction which must vanish on $\delta \mathrm{P}$ and which is defined to be zero in the open region exterior to $P$. It can be shown (see ref. 21) that $u_{1}$ cannot change sign in $P$, so we choose $u_{1} \geq 0$. For each value of $u_{1}$ construct a subregion of $P$ for which $u_{1}$ is greater than some constant $t$, and denote this subregion by $\mathrm{P}_{t}{ }^{\cdot}$ It is assumed $P$ is shaped such that each level curve $u_{1}=t$ is unique and closed, which implies that $P_{t_{1}}$ is a subset of $P_{t_{2}}$ if $t_{1}>t_{2}^{22,23}$. Next construct 
the $\ell$-dimensional hypersphere $E$ with boundary $\delta E$ having the same $\ell$ content or hypervolume as $\mathrm{P}$. We may associate with $\mathrm{E}$ a function $u\left(x_{1} \cdots x_{\ell}\right)$ such that for $0 \leq t \leq \operatorname{Max}_{1}$ the level curve $u=t$ defines a subregion of $E, E_{t}$, having the same $l$ content as $P_{t}$. For convenience, Eq. (A.I) is cast in its equivalent integral form

$$
\begin{gathered}
\lambda_{\mathrm{P}, \ell}^{0}=\operatorname{Min} \mathrm{R}_{\mathrm{P}}, \\
\operatorname{Min} \mathrm{R}_{\mathrm{P}}=\left\lfloor\int_{\mathrm{P}} \cdots \ell \cdot \int_{\mathrm{P}}\left(\sum_{\mathrm{i}=1}^{\ell}\left(\partial \mathrm{u}_{1} / \partial \mathrm{x}_{\mathrm{i}}\right)^{2}\right) \mathrm{dV} / \int_{\mathrm{P}} \cdots \ell \cdot \cdots \int_{\mathrm{P}} \mathrm{u}_{1}{ }^{2} \mathrm{dV}\right] \\
\mathrm{dV}=\mathrm{dx}_{1} \cdots \mathrm{dx}_{\ell}, \\
\left.\mathrm{u}_{1}\right|_{\delta \mathrm{P}}=0 .
\end{gathered}
$$

We will prove that

$$
\begin{aligned}
& \operatorname{Min} R_{P} \geq R_{E} \text {, } \\
& R_{E}=\left[\int_{E} \cdots \ell \cdot \cdots \int_{E}\left(\sum_{i=1}^{\ell}\left(\partial \mathrm{u} / \partial \mathrm{x}_{i}\right)^{2}\right) \mathrm{dV} / \int_{E} \cdots \ell \cdot \cdots \int_{E} \mathrm{u}^{2} \mathrm{dV}\right], \\
& \left.u\right|_{\delta E}=0 .
\end{aligned}
$$


The denominator in $\mathrm{Eq}$. (A.2) may be written

$$
\begin{gathered}
\int_{P} \ldots l \ldots \int_{P} u_{1}^{2} d V=\int_{V(T)}^{V(o)} t^{2} d V(t)=\int_{E} \ldots l \ldots . \int_{E} u^{2} d V \\
T=\operatorname{Max} u_{1},
\end{gathered}
$$

where $V(t)$ indicates the $l$ content of $E_{t}$ and $P_{t}$. Now we determine the relation between the numerators of Min $R_{P}$ and $R_{E}$. First we note that

$$
\left(\nabla_{\sim} \ell_{1}^{\mathrm{u}_{1}}\right) \cdot\left(\nabla_{\sim} \ell_{1}^{\mathrm{u}_{1}}\right)=\left(\frac{\partial \mathrm{u}_{1}}{\partial v}\right)^{2}
$$

where $v$ is measured along the inner normal, $n$, to the curve $u_{1}=t$ (see Fig. 3). Then we convert $\mathrm{dx}_{1} \ldots \mathrm{dx}_{\ell}$ to a more convenient form:

$$
\mathrm{dx}_{1} \ldots \mathrm{dx}_{\ell}=\mathrm{dSd} v=\operatorname{dSdt}\left(\partial \mathrm{u}_{1} / \partial v\right)^{-1}
$$

where dS is an $(\ell-1)$ dimensional surface element ${ }^{24}$.

From Eq. (A.6) and Eq. (A.5) we obtain for the numerator of Eq. $(A .2)$

$$
\begin{gathered}
\int_{P} \ldots \ell \ldots \int_{P}\left[\sum_{i=1}^{\ell}\left(\partial u_{1} / \partial x_{i}\right)^{2}\right] d v=\int_{0}^{T} d t \int_{0}^{L(t)}\left(\partial u_{1} / \partial v\right) d S, \\
L(t)=\int_{0}^{L(t)} d S(t) .
\end{gathered}
$$


We will obtain a lower bound for the $\ell$-dimensional integral in Eq. (A.7) using Schwarz's Inequality and the l-dimensional Isoperimetric Inequality. The latter inequality may be demonstrated as follows: consider the hypersphere $\mathrm{E}$ with boundary $\delta \mathrm{E}$ and region $\mathrm{P}$ with boundary $\delta \mathrm{P}$, both having the same hypervolume $\mathrm{V}$. Determine the content of $\delta E$ as a function, $G_{\delta E}(\ell, V)$, of the hypervolume, $V$, of $E$. Using the calculus of variations ${ }^{25}$ it can be shown that given the content, $\mathrm{L}_{\delta \mathrm{P}}(\ell, V)$, of $\delta \mathrm{P}$,

$$
\mathrm{G}_{\delta \mathrm{E}}(\ell, \mathrm{V}) \leq \mathrm{I}_{\delta \mathrm{P}}(\ell, \mathrm{V}),
$$

the equality sign holding if $\delta \mathrm{P} \equiv \delta \mathrm{E}$.

From Eq. (A.8) we obtain, after dropping the subscripts $\delta \mathrm{E}$ and $\delta \mathrm{P}$,

$$
[L(t)]^{2} \geq G^{2}(\ell, V(t))
$$

while Schwarz's Inequality gives

$$
[L(t)]^{2} \leq \int_{0}^{L(t)} \mathrm{dS}\left(\partial u_{1} / \partial v\right) \int_{0}^{L(t)} \mathrm{dS}\left(\partial u_{1} / \partial v\right)^{-1}
$$

In case the level curves of $\mathrm{P}$ are hyperspheres, $\left(\partial \mathrm{u}_{1} / \partial v\right)$ is constant and Eq. (A.10) is an identity.

From Eq. (A.9) and Eq. (A.10) we have 


$$
G^{2}(l, V(t)) \leq \int_{0}^{L(t)} \mathrm{dS}\left(\partial u_{1} / \partial v\right) \cdot \int_{0}^{L(t)} \mathrm{dS}\left(\partial u_{1} / \partial v\right)^{-1},
$$

and from Eq. (A.6),

$$
\begin{gathered}
V(t)=\int_{t}^{T} d \tau \int_{0}^{L(\tau)} d S\left(\partial u_{1} / \partial v\right)^{-1}, \\
\int_{0}^{L(t)} d S\left(\partial u_{1} / \partial v\right)^{-1}=-\frac{d}{d t} \int_{t}^{T} d \tau \int_{0}^{L(\tau)} d S\left(\partial u_{1} / \partial \nu\right)^{-1}=-\frac{d V(t)}{d t} .
\end{gathered}
$$$$
\text { Using Eq. (A.12), we write Eq. (A.11) as }
$$$$
-G^{2}(l, V(t))\left[\frac{\mathrm{dV}(\mathrm{t})}{\mathrm{dt}}\right]^{-1} \leq \int_{0}^{\mathrm{L}(\mathrm{t})} \mathrm{dS}\left(\frac{\partial \mathrm{u}_{1}}{\partial v}\right)^{26}
$$

and integrate both sides over $t$ from $o$ to $T$ to produce

$$
-\int_{0}^{T} G^{2}(l, V(t))\left[\frac{d V(t)}{d t}\right]^{-1} d t \leq \int_{0}^{T} d t \int_{0}^{L(t)} d S\left(\frac{\partial u_{1}}{\partial v}\right)=\int_{P} \ldots l \ldots \int_{P}\left[\sum_{i=1}^{\ell}\left(\partial u_{1} / \partial x_{i}\right)^{2}\right] d V .
$$

Suppose that in Eq. (A.14) we replace $\mathrm{P}$ with E. This means that Eq. (A.9) and Eq. (A.10) are identities, implying that Eq. (A.11) and Eq. (A.13) are also identities for each $t$. Integrating over $t$ produces the identity form of Eq. (A.14).

Eq. (A.3) has been proved and it remains for us to find a lower bound on $R_{E} \cdot R_{E}$ may be written in hyperspherical coordinates as 


$$
\begin{gathered}
R_{E}=\int_{0}^{r} d r\left[\frac{d R(r)}{d r}\right]^{2} r^{l-1} / \int_{0}^{r} R^{2}(r) r^{l-1} d r, \\
R\left(r_{0}\right)=0,
\end{gathered}
$$

and $r_{0}$ is the radius of hypersphere $E$. Again using the calculus of variations, we find that $M i n R_{E}$ is attained if $R$ satisfies

$$
\begin{aligned}
& \frac{\mathrm{d}^{2} \mathrm{R}(\mathrm{r})}{\mathrm{dr}^{2}}+\frac{(\ell-1)}{\mathrm{r}} \frac{\mathrm{dR}(\mathrm{r})}{\mathrm{dr}}+\lambda_{\mathrm{E}, \ell^{\mathrm{R}}(\mathrm{r})=0,}^{\mathrm{s}}=0, \\
& \mathrm{R}\left(\mathrm{r}_{\mathrm{o}}\right)=0, \\
& \lambda_{\mathrm{E}, \ell}^{\mathrm{O}}=\operatorname{Min} \mathrm{R}_{\mathrm{E}}^{27} .
\end{aligned}
$$

We introduce the change of variable $z=\left(\lambda_{\mathrm{E}, \ell}^{\mathrm{O}}\right)^{\frac{1}{2}} \mathrm{r}$, make the substitution $Z(z)=z^{\alpha} W(z)$ and determine $\alpha$ such that for $\ell$ even, Eq. (A.16) becomes Bessel's Equation for Integral orders ${ }^{28,29}$. $\alpha$ is found to be $(\ell-2) / 2$ and so Eq. (A.16) assumes the form

$$
\begin{aligned}
& z^{-(\ell-2) / 2}\left[z^{2} \frac{d^{2} W(z)}{d z^{2}}+z \frac{d W(z)}{d z}+\left(z^{2}-(\ell-2)^{2} / 4\right) W(z)\right]=0, \\
& W\left(\left(\lambda_{E, \ell}^{O}\right)^{\frac{1}{2}} r_{0}\right)=0,
\end{aligned}
$$

with solutions $W(z)=J_{\frac{\ell-2}{2}}(z)^{30}, \frac{J^{\ell-2}}{2}(z)$ being the Bessel Function of order $(\ell-2) / 2$. 
Our final result is

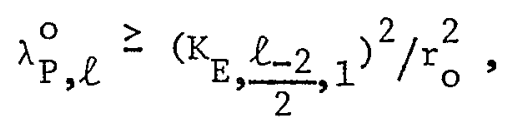

where $\mathrm{K}_{\mathrm{E}, i, j}$ indicates the $j$ th root of the Bessel Function $J_{i}(x)$. In terms of the lowest energy level of the $(\ell / d)$-particle system at high density we have

$$
\begin{aligned}
& \mathrm{E}_{\mathrm{P}, \ell}^{\mathrm{O}} \geq \frac{\hbar^{2}}{2 \mathrm{~m}} \mathrm{~K}_{\mathrm{E}, \frac{\ell-2}{2}, 1 / \mathrm{r}_{\mathrm{o}}^{2}(\mathrm{~V}),} \\
& \mathrm{E}_{\mathrm{P}, \ell}^{\mathrm{O}} \geq \frac{\hbar^{2}}{2 \mathrm{~m}} \mathrm{~K}_{\mathrm{E}, \frac{\ell-2}{2}, 1}^{2} \pi /\left(\mathrm{V} \Gamma\left(1+\frac{\ell}{2}\right)\right)^{\frac{2}{\ell}}, 31
\end{aligned}
$$

the equality holding only if $\mathrm{P}$ is the hypersphere E. In Eq. (A.19) $\Gamma(x)$ denotes the Gamma Function of argument $x$. 
APPENDIX B

In this section we prove that linear boundary conditions give the correct high density limit for each eigenvalue (and consequently the Helmholtz Free Energy) associated with the cluster $(n, t)$. The proof is valid for hard disks and hard sphere systems.

Consider the region $P$ of accessible configurations whose boundary $\delta P$ is composed of pieces of surfaces $S(i, j)$ where $i$ and $j$ denote a nearest neighbor pair in $(n, t)$. For each surface $s(i, j)$ we define the point $D(i, j)$ of closest approach to the origin, the origin being the lattice site of particle 1.

First consider polytope $\mathrm{P}_{1}$, its boundary $\delta \mathrm{P}_{1}$ consisting of the hyperplanes tangent to $S(i, j)$ at $D(i, j)$ for each nearest neighbor pair in $(n, t)$. Next consider a polytope $P_{2}$ which is geometrically similar to $P_{1}$ and which is oriented so that if its hypervolume were suitably changed (its geometry remaining the same) $\mathrm{P}_{2}$ would be coincident with $\mathrm{P}_{1}$. We may adjust the volume of $\mathrm{P}_{2}$ so that $\mathrm{PCP}$ and at least one point of $\delta \mathrm{P}$ is on $\delta_{2}$. Choose one such point and let the vector from the origin to this point be $\mathrm{d}_{2} \mathrm{u}$, where $\mathrm{u}$ is a unit vector. Let the corresponding vector from the origin to $\delta \mathrm{P}_{1}$ be $\mathrm{d}_{1} \mathrm{u}$ (see Fig. 5).

Salsburg et al. ${ }^{32}$ has shown that

$$
\mathrm{d}_{2}^{\mathrm{u}} \underset{\mathrm{v} \rightarrow \mathrm{v}_{0}}{=}\left(\mathrm{d}_{1}+o\left(\frac{\mathrm{v}}{\mathrm{v}_{\mathrm{o}}}-1\right)^{2}\right)_{\sim}^{\mathrm{u}},
$$

$v$ being the specific volume and $v_{0}$ the close packed specific volume. 
Corresponding to the set inequality

$$
\mathrm{P}_{1} \subset \mathrm{P} \subset \mathrm{P}_{2}
$$

we have the eigenvalue inequality

$$
\lambda_{P_{2}}^{j} \leq \lambda_{P}^{j} \leq \lambda_{P_{1}}^{j}, \quad 33 \quad j=0,1,2,3 \ldots
$$

where

$$
\begin{aligned}
& \lambda_{\mathrm{P}_{1}}^{\mathrm{j}}=\frac{\mathrm{K}^{j_{\hbar}^{2}}}{2 \mathrm{~m} \mathrm{~d}_{1}^{2}}, \\
& \lambda_{\mathrm{P}_{2}}^{\mathrm{j}}=\frac{\mathrm{K}_{\mathrm{h}}^{j^{2}}}{2 \mathrm{~m} \mathrm{~d}_{2}^{2}}
\end{aligned}
$$

From Eq. (B.1) we have

$$
\mathrm{d}_{2}^{-2}=\mathrm{d}_{1}^{-2}\left[1+0\left[\frac{\left(\frac{\mathrm{v}}{\mathrm{v}}-1\right)^{2}}{\mathrm{~d}_{1}}\right]\right] \text {. }
$$

$$
\begin{aligned}
& \text { Now } \mathrm{d}_{1}=\mathrm{c}_{0}(\mathrm{a}-\sigma) \text {, where } \mathrm{c}_{0} \text { is a constant, and } \\
& \frac{\mathrm{v}}{\mathrm{v}_{0}}-1=\frac{1}{\sigma^{2}}\left(\mathrm{a}^{2}-\sigma^{2}\right) \underset{\mathrm{d}_{1} \rightarrow 0}{\longrightarrow} \frac{2 \mathrm{~d}_{1}}{\sigma \mathrm{c}_{0}},
\end{aligned}
$$

so

$$
\mathrm{d}_{2}^{-2} \underset{\mathrm{d}_{1} \rightarrow 0}{=} \mathrm{d}_{1}^{-2}\left[1+o\left(\mathrm{~d}_{1}\right)\right]
$$


and

$$
\lambda_{P_{1}}^{j}\left[1+0\left(d_{1}\right)\right] \leq \lambda_{P}^{j} \leq \lambda_{P_{1}}^{j}
$$

As $d_{1} \rightarrow 0 \quad \lambda_{P}^{j}$ asymptotically approaches $\lambda_{P_{1}^{j}}^{j}$.

For a concrete example of Eq. (B.3), let us consider the single particle cell for hard disks in a triangular lattice (see Fig. 2 and Fig. 6). The upper and lower bounds on $\lambda_{\mathrm{P}}^{j}$ are, respectively

$$
\begin{aligned}
& \lambda_{\mathrm{P}_{1}}^{\mathrm{j}}=\frac{\mathrm{k}_{\mathrm{h}^{2}}^{\mathrm{j}^{2}}}{2 \mathrm{m \textrm {d } _ { 1 }}{ }^{2}}, \\
& \lambda_{\mathrm{P}_{2}}^{\mathrm{j}}=\frac{\mathrm{k}_{\mathrm{h}^{2}}^{\mathrm{j}^{2}}}{2 \mathrm{md_{2 }}},
\end{aligned}
$$

where $d_{1}$ and $d_{2}$ are shown in Fig. 6. From the equations of the two circles at $(a / 2,(\sqrt{3} / 2) a)$ and $(a, 0)$, we have from their intersection

$$
d_{2}=\frac{3}{4} a-\frac{1}{2}\left(9 a^{2}-24 a d_{1}+12 d_{1}^{2}\right)^{\frac{1}{2}}
$$

We expand Eq. (B.10) in a Taylor Series about $d_{1}=0$,

$$
\mathrm{d}_{2}=\mathrm{d}_{1} \pm \frac{1}{6} \frac{\mathrm{d}_{1}^{2}}{\mathrm{a}}+\frac{2}{9} \frac{\mathrm{d}_{1}^{3}}{\mathrm{a}^{2}}+0\left(\frac{\mathrm{d}_{1}^{4}}{\mathrm{a}^{3}}\right) \text {, }
$$

so that

$$
\mathrm{d}_{2}^{-2}=\mathrm{d}_{1}^{-2}\left[1+\frac{1}{3} \frac{\mathrm{d}_{1}}{\mathrm{a}}+\frac{17 \mathrm{~d}_{1}^{2}}{16 \mathrm{a}^{2}}+0\left(\frac{\mathrm{d}_{1}^{3}}{\mathrm{a}^{3}}\right)\right]^{-1},
$$


or

$$
d_{2}^{-2}=d_{1}^{-2}\left[1-\frac{1}{3} \frac{d_{1}}{a}-\frac{13}{36} \frac{d_{1}^{2}}{a^{2}}+0\left(\frac{d_{1}^{3}}{a^{3}}\right)\right]
$$

Eq. (B.3) then becomes

$$
\frac{\mathrm{K}^{j_{\hbar^{2}}}}{2 \mathrm{~m} \mathrm{~d}_{1}^{2}}\left[1-\frac{1}{3} \frac{\mathrm{d}_{1}}{\mathrm{a}}-\frac{13}{36} \frac{\mathrm{d}_{1}^{2}}{\mathrm{a}^{2}}+\mathrm{O}\left(\frac{\mathrm{d}_{1}^{3}}{\mathrm{a}^{3}}\right)\right] \leq \lambda_{\mathrm{P}}^{\mathrm{j}} \leq \frac{\mathrm{K}_{\mathrm{h}^{2}}^{{ }^{2}}}{2 \mathrm{~m} \mathrm{~d}_{1}^{2}}, \quad \text { (B.14) }
$$

and as $\mathrm{d}_{1} \rightarrow 0$ the lower bound asymptotically approaches the upper bound. 
TABLE $\mathrm{I}$. The first 20 eigenvalues for the single disk cell are given in units of $\hbar^{2} /\left(2 m(a-\sigma)^{2}\right.$, where $2 \pi \hbar=h$ is Planck's Constant, $m$ is the mass of a disk, $a$ is the nearest neighbor lattice spacing and $\sigma$ is the diameter of a disk

\begin{tabular}{|l|l|l|l|}
\hline 5.3667 & 28.120 & 52.609 & 70.752 \\
13.599 & 35.727 & 52.609 & 70.752 \\
13.599 & 39.478 & 65.652 & 82.789 \\
24.340 & 45.083 & 65.652 & 90.661 \\
24.340 & 45.083 & 67.551 & 92.118 \\
\hline
\end{tabular}

IABLE II. The first 20 eigenvalues for the nearest neighbor pair of disks are in units of $\hbar^{2} /\left(2 m(a-\sigma)^{2}\right.$, where $2 \pi \hbar=h$ is Planck's Constant, $m$ is the mass of a single disk, $a$ is the nearest neighbor lattice spacing and $\sigma$ is the disk diameter.

\begin{tabular}{|c|c|c|c|}
\hline 11.360 & 26.391 & 31.669 & 37.662 \\
18.352 & 27.818 & 31.669 & 38.045 \\
19.615 & 27.842 & 32.523 & 39.193 \\
19.615 & 27.842 & 33.250 & 41.318 \\
21.504 & 29.884 & 35.385 & 41.318 \\
\hline
\end{tabular}


TABLE III. The lowest eigenvalues for two types of hard sphere single cells are given in units of $h^{2} /\left(2 \mathrm{~m}(a-\sigma)^{2}\right)$, where $2 \pi h=h$ is Planck's Constant, $m$ is the mass of a sphere, a is the nearest neighbor lattice spacing and $\sigma$ is the sphere diameter. The volumes of the two cells are identical so the classical Helmholtz Free Energy in the single particle free volume approximation is the same for both. The quantum calculations (neglecting correlations) predict the hard sphere h.c.p. lattice to be the stable configuration at high pressures and low temperatures.

\begin{tabular}{|c|c|}
\hline $\begin{array}{c}\text { f.c.c. } \\
\text { (rhombic dodecahedral cel1) }\end{array}$ & $\begin{array}{c}\text { h.c.p. } \\
\text { (icosahedral cel1) }\end{array}$ \\
\hline 8.4970 & 8.4969 \\
17.298 & 17.293 \\
17.298 & 17.293 \\
17.298 & 17.299 \\
\hline
\end{tabular}


Fig. 1. A two dimensional outline of the accessible configurations of the nearest neighbor pair of disks in a triangular lattice. The disk on the right hand side is fixed at an arbitrary position which determines the accessible region for the disk on the left hand side. 

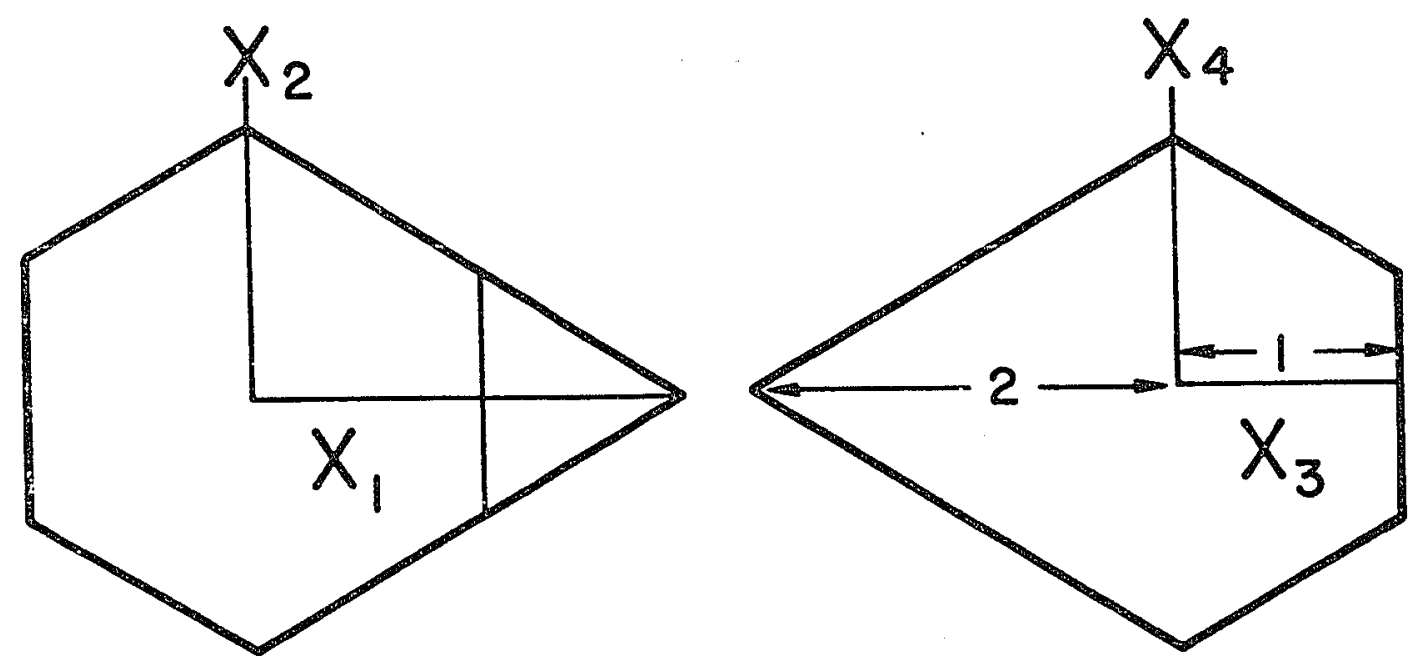
Fig. 2. A triangular array of disks is shown. The single particle cell is bounded by circles of radius $\sigma$ centered on nearest neighbor lattice sites... 


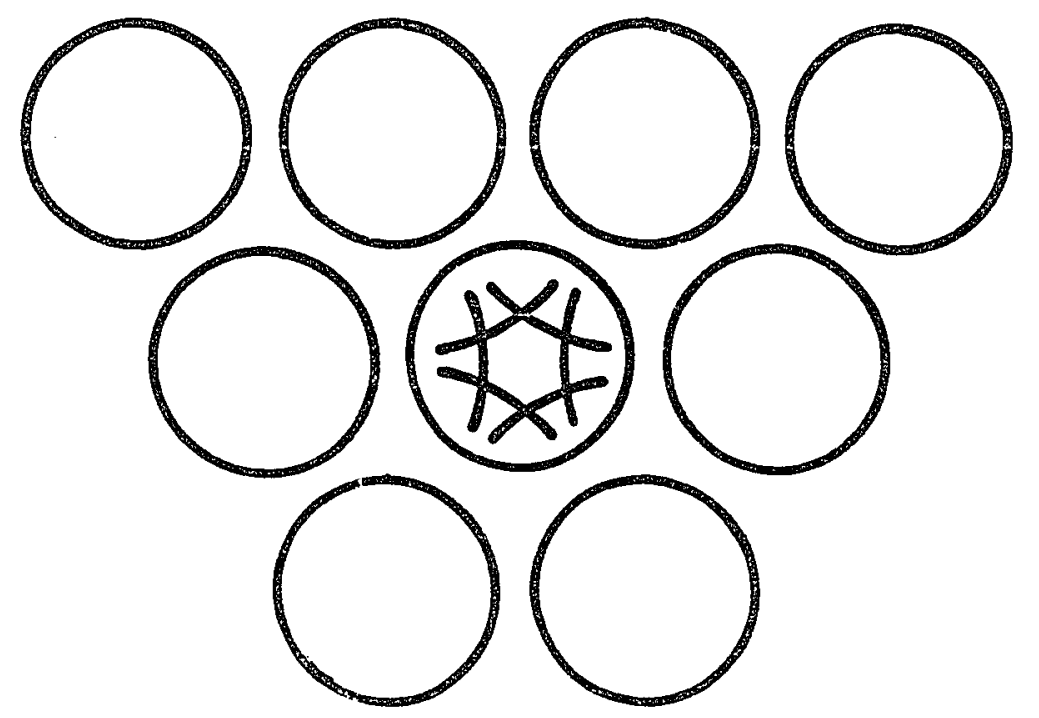


Fig. 3. The classical and quantum approximations to $\mathrm{F} / \mathrm{Nk}_{B} \mathrm{~T}$, where $\mathrm{F} / \mathrm{N}$ is the Helmholtz Free Energy per particle and $k_{B} \mathrm{~T}$ is Boltzmann's Constant times the absolute temperature, are plotted against $B$, which is defined as $B=h^{2} /\left(\sigma^{2} 2 \pi^{2} m k_{B} T\left(\frac{V}{v_{o}}-1\right)^{2}\right)$. $\mathrm{h}$ is Planck's Constant, $\sigma$ is the disk diameter, $\mathrm{m}$ is the mass of a disk, $v$ is the specific volume and $v_{0}$ the close packed specific volume. 


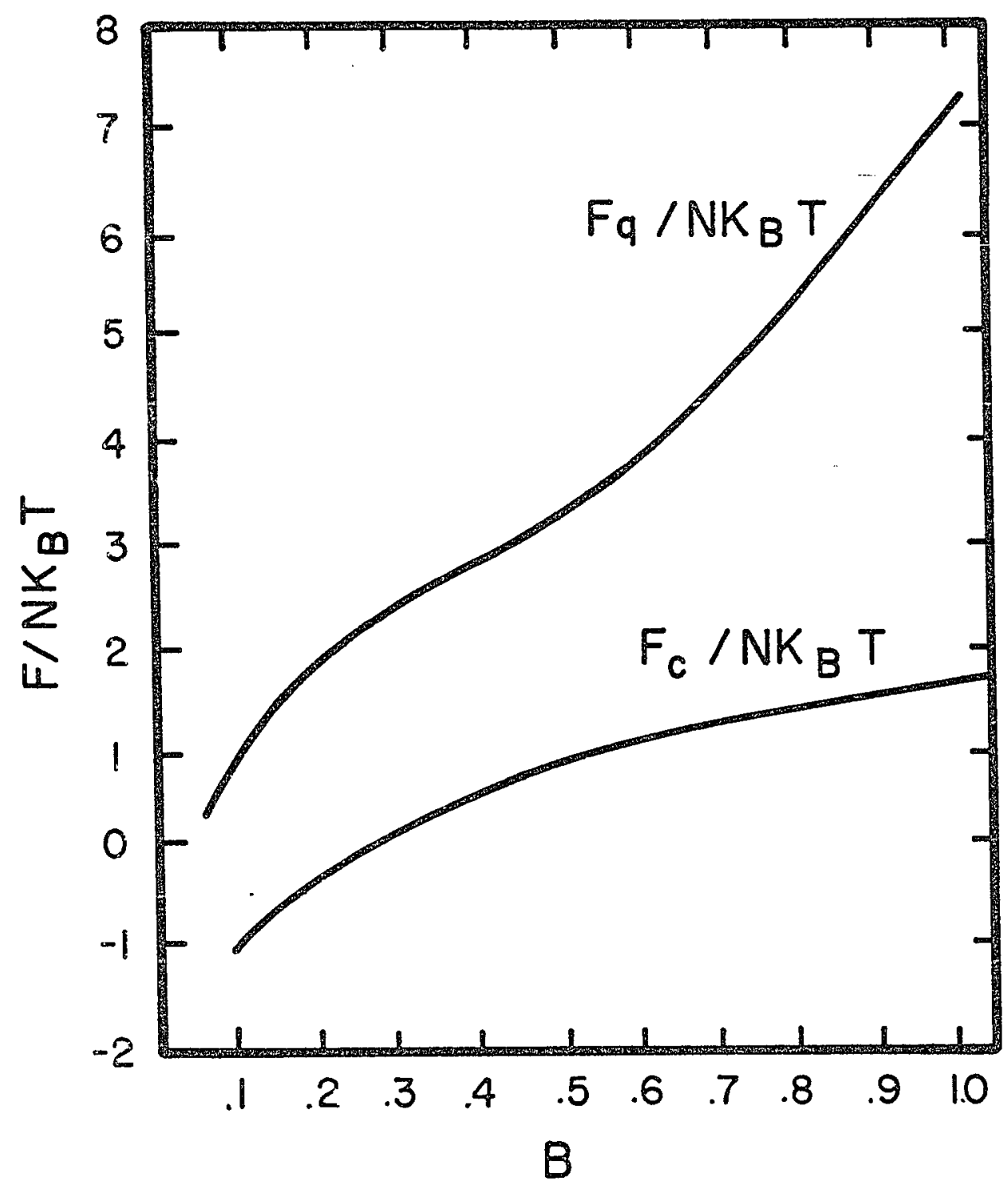


Fig. 4. For $l=2$, region $P$ is represented with three possible inner normals from its boundary. The inner normals are perpendicular to each level curve $u_{1}=t$ at the point of intersection with the level curve. 


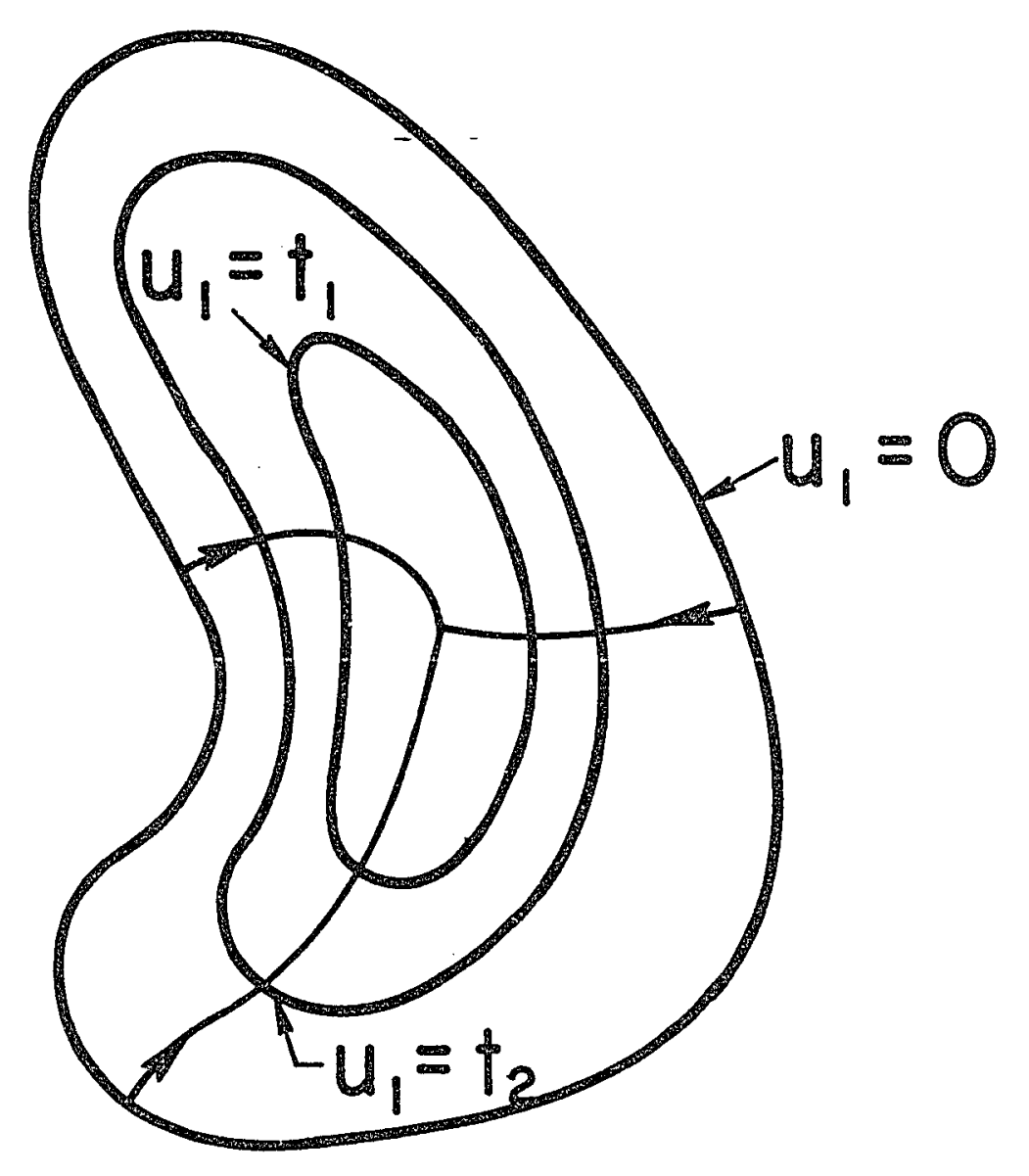


Fig. 5. The region $P$ of accessible configurations is shown with polytopes $\mathrm{P}_{1}$ and $\mathrm{P}_{2}$ where $\mathrm{P}_{1} \subset \mathrm{P} \subset \mathrm{P}_{2}$ with $\mathrm{P}_{2}$ geometrically similar to $\mathrm{P}_{1}$. The distance measured along $\underset{\sim}{u}$ from the origin to $\delta \mathrm{P}_{1}$ is $\mathrm{d}_{1}$, and the distance along $\mathrm{u}$ from the origin to $\delta \mathrm{P}$ is $\mathrm{d}_{2}$. In this drawing $\delta \mathrm{P}$ and $\delta \mathrm{P}_{2}$ have one point in common. 


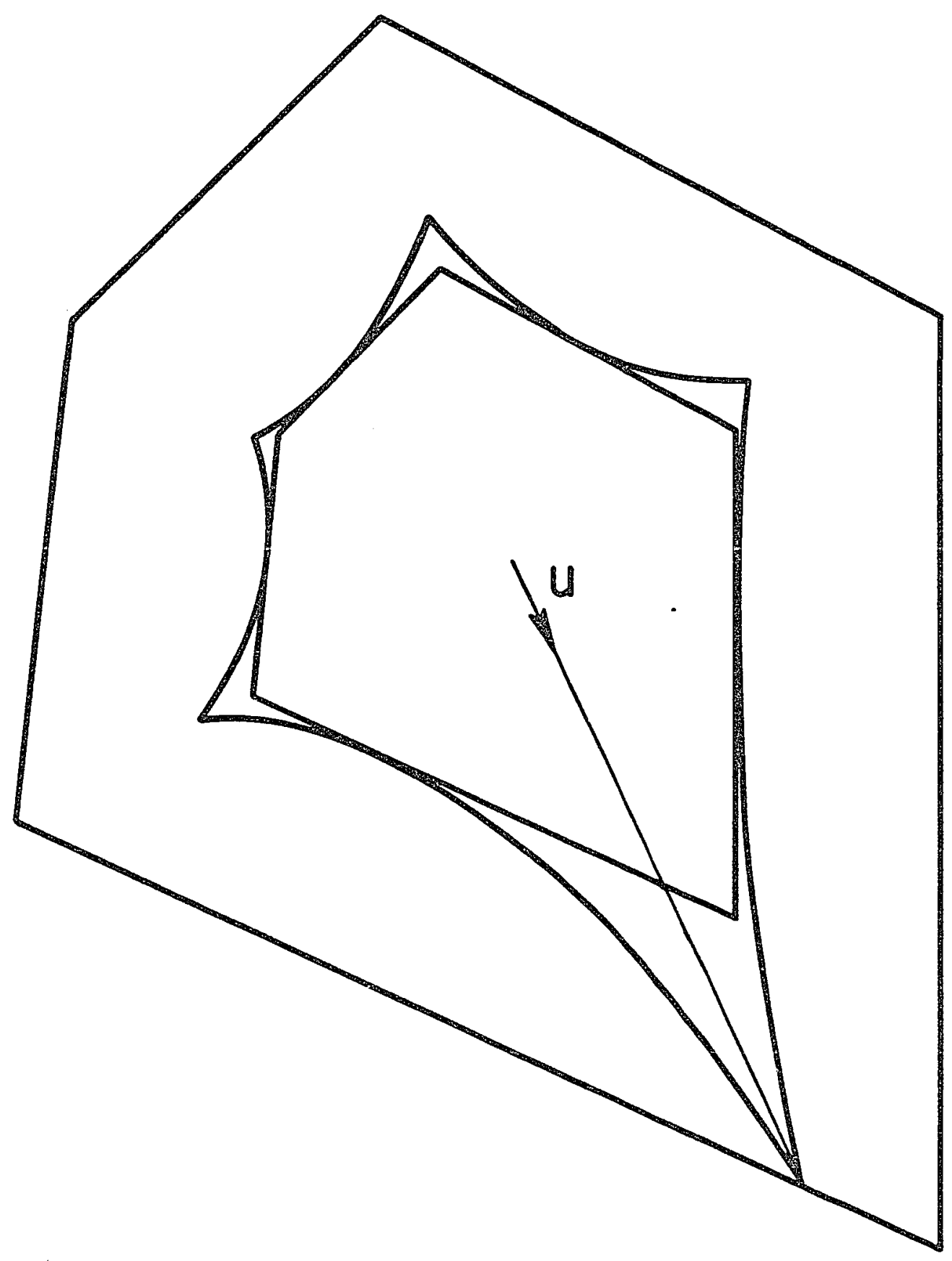


Fig. 6. The single particle cell for disks in a triangular lattice is shown (region $P$ ) with the inscribed hexagon $P_{1}$ and the circumscribed hexagon $\mathrm{P}_{2}$. 


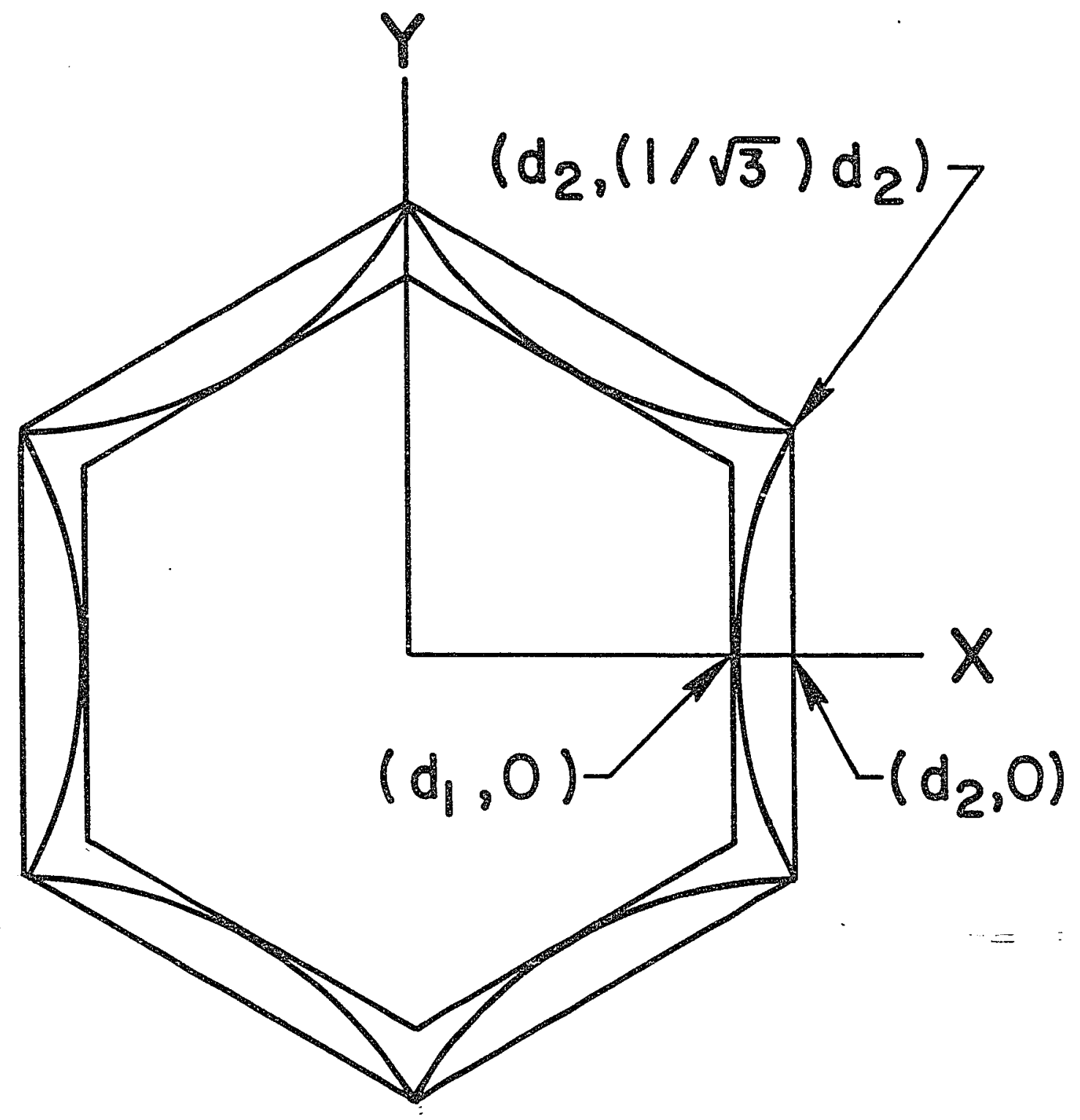




\section{REFERENCES}

1. C. N. Yang, Physica 26, 549 (1960).

2. B. Alder, B. Carter, D. Young (private communication; to be published).

3. For a more detailed discussion of the cell cluster formalism, see Z. Salsburg, W. Rudd, F. Stillinger, R. Kornegay, 予. Chem. Phys. 43, 932 (1965).

4. Two subclusters of type $(\ell, S)$ are counted separately if they have at least one point that is not common to both.

5. This equivalence is by no means obvious, and it may not be true in the thermodynamic limit. For a proof of equivalence for finite $N$, see ref. 3 .

6. Z. Salsburg, W. Rudd, F. Stillinger, J. Chem. Phys. 47, 4534 (1967).

7. For an involved discussion of a perturbation technique which employs the Green function for a polytope containing the polytope considered, see Morse and Feshbach, "Methods of Theoretical Physics" (McGraw-Hill, New York, 1953), Vo1. II, page 1060.

8. It is possible to establish upper and lower bounds on the Kth eigenvalue for the region $P$ if one is willing to evaluate $\int_{\mathrm{P}} \psi_{\mathrm{K}} \nabla^{4} \psi_{\mathrm{K}} \mathrm{d} \tau$. See D. H. Weinstein, Proc. Nat.Acad. Sci, $\underline{20}, 529$ (1934); see also J. K. L. Macdonald, Phys. Rev. 46, 828 (1934). 
9. See ref. 2 .

10. See ref. 6 .

11. R. K. Cole, Phys. Rev. 155, 114 (1967).

12. Z. Salsburg, W. Rudd, A. Yu, F. Stillinger, J. Chem. Phys., 49, 4857 (1968).

13. The constants $\gamma_{0}$ and $\gamma_{1}$ are not known exactly, but because the series for $\gamma_{0}$ and $\gamma_{1}$ seem to converge rapidly, the values given should be accurate enough for our purposes.

14. It should be noted that since we are evaluating an asymptotic function $\mathrm{E}_{\mathrm{O}}(\rho)$ at a given density, a function which is asymptotically equivalent to $E_{0}(\rho)$ may give quite different results at this density.

15. The simple cubic packing of hard spheres gives $A=\pi^{2}$, an exact result, See ref. 11 .

16. Z. W. Salsburg, J. Chem. Phys. 44, 3752 (1966).

17. W. G. Hoover, J. Chem. Phys. 43, 371 (1965).

18. The convex properties of the Helmholtz Free Energy may be used to establish upper and lower bounds on the pressure exerted by quantum hard spheres at $0^{\circ} \mathrm{K}$. For a general discussion of these properties see M. E. Fisher, J. Chem. Phys. 42, 3852, (1965).

19. An interesting presentation of the theory of solid $\mathrm{He}^{3}$ is given by L. H. Nosonow, Phys. Rev. Letters, 13, 270 (1964). 
20. For a discussion of spin alignment in solid $\mathrm{He}^{3}$ see Bernardes and Primakoff, Phys. Rev. 119.1, 968 (1960).

21. P. Garabedian, "Partial Differential Equations," (John Wiley and Sons, New York, 1964), Chap. 11.

22. J. Dugundji, "Topology," (Allyn and Bacon, Boston, 1966), Chap. 5.

23. It seems to be a difficult topological problem to determine the restrictions on the shape of $\mathrm{P}$ such that $\mathrm{u}_{1}$ has level curves which are unique and closed. Garabedian (see ref.21) indicates that any closed, simply connected region will suffice for $l=2$, but he offers no proof of this.

24. A. Erdélyi, "Higher Transcendental Functions," (McGraw-Hill, New York, 1953), Vo1. II, Chap. 11.

25. H. Margenau and G. Murphy, "The Mathematics of Physics and Chemistry," (D. Van Nostrand Co., New York, 1956), Vol. I, Chap. 6.

26. Since $d v(t) / d t$ is a negative quantity, the direction of the inequality is not changed when we divide both sides by $-(d v(t) / d t)$.

27. In Eq. (A.15), substitute $R(r)=R(r)+\varepsilon h(r)$ and using the criterion for Min $R_{E}$ to be attained, that ( $\partial$ Min $\left.R_{E} / \partial \varepsilon\right)_{\varepsilon=0}=0$, the differential equation $\mathrm{Eq}$. (A.16) may be derived.

28. For $l$ odd, Bessel Functions of Fractional Order are the solutions to Eq. (A.16). 
29. M. Abramowitz and I. Stegun, "Handbook of Mathematical Functions," (Nat1. Bureau of Standards, 1966), Chap. 9.

30. The actual solution of Eq. (A.17) is $S(z)=z^{-\frac{(\ell-2)}{2}} \frac{\mathrm{J}-2}{2}(z)$, the zeros of $S(z)$ being determined by the zeros of

-.. $\frac{J_{\ell-2}}{2}(z) . S(z)$ remains finite and non-zero at the origin, since $\lim S(z)=2^{-(\ell-2) / 2}[(\ell / 2-1) !]^{-1}$. It can also be shown that $S(z)$ $z \rightarrow 0$

is a monotonically decreasing function in the interval $\left[0,\left(\lambda_{\mathrm{E}, \ell}^{0}\right)^{\frac{1}{2}} \mathrm{r}_{0}\right]$, which implies that each level curve $S(z)=t$ is unique and closed.

31. Sommerville, "The Geometry of N Dimensions," (Dover, New York, 1958), Page 136.

32. Z. W. Salsburg and W. W. Wood, J. Chem. Phys. 37, 798 (1962)。

33. For a proof of this inequality see Courant and Hilbert, "Methods of Mathematical Physics," (Interscience, New York, 1966), Vol. I, Chap VI. 